\title{
Novel Approaches to Improving the Quality of the Clinical Trial System through a Directional Comparative Study
}

\author{
Tenzin Kunga *, Masataka Sano *, Motoi Iwashita *
}

\begin{abstract}
This article compares and analyzes the correlation between the GCP Integrated AddendumICH E6 (R2) protocols and Quality Management System (QMS) ISO 9001:2015 requirements for improving the clinical trials system. A comparative analysis is a directional approach, created from the clinical trial guideline (ICH E6 (R2)-addendum) toward the ISO 9001:2015 quality standards. This directional perspective will allow a more accurate comparison and help identify any gaps that might exist in order to achieve quality performance in clinical trial practice. This paper concludes that the addendum mainly focuses on the risk-based approach. This approach, a perspective from the clinical trials and quality management system, can examine and find novel ways to improve the efficiency of the clinical trial system. This study shows a directional perspective on and comparative analysis between the clinical trial guidelines (ICH E6 (R2)-addendum) and the ISO 9001:2015 quality standards in this domain, there is no prior research in this direction for Addendum.
\end{abstract}

Keywords: Addendum, Clinical Trials, International Standard Organization, Quality Management System, International Council for Harmonization, Risk-Based Approach

\section{Introduction}

With improvement in the field of medical research and technology; quality performance becomes an important part of the pharmaceutical companies. In the healthcare sector, clinical trials are a crucially important part of the R\&D process. Now, the pharmaceutical companies are shifting marketplace from product-driven concept to customer satisfaction.

\subsection{Good Clinical Practice (GCP)}

The International Conference on Harmonization (ICH) GCP document (referred to as GCP) is defined as GCP deals with the scientific quality standards for designing, conducting, recording, and reporting trials that involve the participation of human subjects [1].

\footnotetext{
* Chiba Institute of Technology, Chiba, Japan
} 
The objective of this ICH GCP Guideline is to provide a unified standard to facilitate the mutual acceptance of clinical data by the regulatory authorities of the European Union (EU), Japan, and the United States [1]. The goal is to provide updated guidance that is both appropriate and flexible enough to address the increasing diversity of clinical trial designs and data sources that are being employed to support regulatory and other health policy decisions [5]. The underlying principles of human subject protection and data quality should remain.

In the addendum, the amendments in several sections-glossary, principles, sponsor responsibilities, investigator responsibilities, and essential documents-reflect new approaches and systems with an emphasis on human subject protection and data integrity [1]. A certified copy is a copy (irrespective of the type of media used) of the original record that has been verified (e.g., by a dated signature or by generation through a validated process) to have the same information as the original, including data that describe the context, content, and structure [1].

In the event of any conflict between the ICH E6 (R1) text and the E6 (R2) addendum text, the E6 (R2) addendum text should take priority [1]. Therefore, this research paper mainly focused on addendum.

\subsection{Quality Management System (QMS)}

The sponsor should implement a quality management system (QMS) that focuses on trial activities essential to ensuring human subject protection and reliability of trial results. The QMS includes the design of well-organized protocols, tools, and processes for data collection and management, and the collection of all information that is crucial to decision-making [1].

To have a quality management system is a strategic decision for an organization that can help improve its overall performance and provide a sound basis for sustainable development initiatives [2]. The quality management system should function per international standard regulations. Pharmaceutical companies in the pursuit of excellence, they followed the path of achieving a quality certification; namely, ISO 9000 accreditation [7]. We can use ISO 9001:2015 as the based system to certification and stepping-stone on the journey for seeking continuous improvements.

The quality management system at a trial site, which is an important element of the clinical stage of drug development, can be easily integrated into the entire system of quality assurance that embraces all stages of the drug lifecycle [3].

\subsection{GCP and QMS relations}

The GCP approach is relative to quality standards. But it does not define what is meant by a quality management system (QMS) and because of this, quality assurance (QA) systems may be based on the implementation of standard operating procedures (SOPs). SOPs are an important part of a quality system, but a collection of SOPs does not equal a quality system [3].

The purpose of this research is to study the detail analysis and comparison of the ISO 9001: 2015 quality management methodology and GCP requirements to elaborate the methodological background in the context of this consistent integration for enhancing the clinical trials system [4]. But in this paper, they consider only comparative analysis. Thus, the directional approach, created from the clinical trials guideline (ICH-E6 (R2)-addendum) 
toward the ISO 9001:2015 quality standards might allow a more accurate comparison between them and help identify any gaps that might exist to achieve quality performance in clinical trials system.

\section{Objective of the Article}

A. Directional approach-Comparative analysis of Integrated Addendum to ICH E6 (R1): Guideline for GCP E6 (R2) toward the ISO 9001: 2015.

B. More specifically, a direct correlation between the GCP Integrated Addendum- ICH E6 (R2) protocols and the QMS ISO 9001:2015 requirements for improving the clinical trial system.

The aim of this paper is as mentions above; by following the directional approach of comparative analysis between them, this might give a broad perspective from GCP E6 (R2) toward QMS. By finding the correlation between them will give a road map to follow the path for improving the quality of GCP guidelines.

The second objective is to find the similarity between the sentence-to-sentence/word-toword of the GCP Integrated Addendum-ICH-E6 (R2) protocols and the QMS ISO 9001:2015.

\section{Protocol}

In the research paper, GCP guidelines and ISO both considered the importance of measure, analysis, and improvement. GCP advocates the amendments process, for regulatory applications and documents, while ISO focuses on corrective and preventive actions [3]. It shows how contract document control and corrective action are the main themes to both ISO and GCP. For example, ISO outlines the need for document control, as documents must be approved before use [6]. GCP is designed to execute the clinical practices that involve the participation of human subjects. In the GCP method, it mainly focuses on regulation and documentation of SOPs, protocol, and responsibilities involves sponsors and investigators.

This paper reviews the addendum to the ICH E6 (R1): the guideline for the Good Clinical Practice E6 (R2). Good clinical practice has different stages of clinical trials that have more detailed procedures. In addition, there are interested parties involved in the process. This addendum mainly covers the risk-based approach and details the monitoring procedures. Generally, quality management standards are applied to the routine practice of clinical trials. The comparative analysis in this paper is directional, and it provides a word-for-word comparison between the GCP addendum, including sponsors and monitoring plans, and the QMS ISO 9001:2015.

This paper is studying the comparative analysis between the GCP Integrated Addendum ICH-E6 (R2) protocols and the ISO 9001:2015. For example, in ICH-E6 (R2) addendum, it says 5.0.2 identify critical processes and data while in the QMS; we have found a similar conceptual meaning, $4.4(f)$ addresses the risks and opportunities that need to be addressed to prevent, or reduce undesirable effects.

This analysis, with the assistance of the Quality Management System, will help us develop new approaches. The new version of ISO 9001: 2015 contains updated information and more details about risk management. Thus, this approach becomes a crucial part of a quality 
management system analysis. As a result, implementing the risk-based approach has provided an opportunity to identify preventive and corrective actions.

\section{Methodology for comparative study;}

Before initiating a comparative study, the objective of analysis must be understood and documented. In the clinical process, the objective is to improve the quality of the clinical trial system that would have a significant impact on the human subject protection, reliability of trial results and compliance with requirements, etc.

By considering the above, the GCP protocols and ISO 9001:2015 requirements should be well understood before proceeding for the comparative study. The document review, document control, and corrective action are essential for both GCP protocols and ISO 9001:2015. Also, analysis and improvement are important for both GCP advocates the amendment process, for regulatory applications and documents, while ISO focuses on corrective and preventive actions [3].

First, the methodology for initiating a comparative study is by selecting the document for the analysis. In GCP Integrated Addendum ICH-E6(R2) protocols, the ICH-E6(R2) addendum was considered as the main document for comparative study. After a thorough understanding of addendum protocols, the ISO 9001:2015 protocol was implemented for comparative study.

Second, considering the directional approach of comparative study. Here, the ICH-E6(R2) addendum texts in a sequential-wise compared with the QMS ISO 9001:2015 requirements. This study involves the participation of several candidates' documents from QMS ISO 9001:2015. The final candidate is selected based on the most relevant information as a minimum variation (author's viewpoint).

Third, for more concrete directional comparative study through direct correlation, there are two sub-group as follow;

1. sentence-sentence correlation, which focuses on the conceptual understanding of the sentences in between these two systems.

2. word-to-word correlation, which considers the direct comparison between the words and finds the closest terminology that can connect these two systems.

Table 1 presents a comparison between the GCP Integrated Addendum ICH-E6(R2) protocols and the QMS ISO 9001:2015 and this table shows the research outcome of their analysis and comparative between them. 
TABLE NO: 1 - COMPARATIVE STUDY BETWEEN THE INTEGRATED ADDENDUM TO ICHE6(R2) AND QUALITY MANAGEMENT SYSTEM (ISO 9001:2015)

\begin{tabular}{|c|c|}
\hline Integrated Addendum to ICH-E6 (R2) & Quality Management System (ISO 9001:2015) \\
\hline \multicolumn{2}{|l|}{ 5. SPONSOR } \\
\hline $\begin{array}{l}5.0 \text { Quality management should focus on essential } \\
\text { trial activities. }\end{array}$ & 4.4 The quality management system \\
\hline $\begin{array}{l}\text { RISK-BASED APPROACH } \\
\text { 5.0.1 Critical Process and Data Identification } \\
\text { - The sponsor should identify critical processes and } \\
\text { data to ensure human subject protection and the } \\
\text { reliability of trial results. }\end{array}$ & $\begin{array}{l}\text { 4.4. Quality Management System } \\
\text { (c) Determine and apply the criteria and methods } \\
\text { necessary to ensure the effective operation and } \\
\text { control of these processes }\end{array}$ \\
\hline $\begin{array}{l}\text { 5.0.2 Risk Identification } \\
\text { - The sponsor should identify risks related to the } \\
\text { critical trial processes and data. }\end{array}$ & $\begin{array}{l}\text { 4.4 Quality Management System } \\
(f) \text { Address the risks and opportunities as determined } \\
\text { in accordance with the requirements. }\end{array}$ \\
\hline $\begin{array}{l}\text { 5.0.3 Risk Evaluation } \\
\text { - The sponsor should evaluate the identified risks } \\
\text { against the existing risk controls. } \\
\text { - The sponsor should evaluate the identified risks, } \\
\text { against existing risk control by considering. }\end{array}$ & $\begin{array}{l}\text { 9.1.3 Analysis and evaluation } \\
\text { - The organization shall analyze and evaluate } \\
\text { appropriate information arising from monitoring or } \\
\text { measurement. } \\
\text { (e) The effectiveness of actions taken to address risks. }\end{array}$ \\
\hline $\begin{array}{l}\text { 5.0.4 Risk Control } \\
\text { - The sponsor should decide which risks to reduce } \\
\text { and/or accept. The approach used to reduce risk to } \\
\text { an acceptable level should be proportionate to the } \\
\text { significance of the risk. } \\
\text { - Protocol design and implementation } \\
\text { - } \text { Agreement between parties } \\
\text { - } \text { Systematic safeguards }\end{array}$ & $\begin{array}{l}\text { 8.3.2 Design and Development Planning } \\
\text { - Determine the stages and controls for design and } \\
\text { development. } \\
\text { The organization shall consider: } \\
\text { (b) } \text { Requirement process stages } \\
\text { (d) } \underline{\text { Responsibilities }} \\
\text { (f) } \underline{\text { Control interfaces }}\end{array}$ \\
\hline $\begin{array}{l}\text { 5.0.5 Risk Communication } \\
\text { Communicate quality management activities to those } \\
\text { who are involved in/affected by such activities. }\end{array}$ & $\begin{array}{l}\text { 6.2 Quality Objectives and Planning to Achieve them. } \\
\text { 6.2.1(c) Quality objectives shall take into account } \\
\text { applicable requirements. } \\
\text { f) Be communicative. }\end{array}$ \\
\hline $\begin{array}{l}\text { 5.0.6 Risk Review } \\
\text { - The sponsor should periodically review the risk } \\
\text { control measures to ascertain whether the } \\
\text { implemented quality management activities remain } \\
\text { effective and relevant, taking into account emerging } \\
\text { knowledge and experience. }\end{array}$ & $\begin{array}{l}\text { 9.3 Management Review: } \\
\text { - The management review shall be planned and carried } \\
\text { out taking into consideration: } \\
\text { Information on the performance and effectiveness of } \\
\text { the QMS. }\end{array}$ \\
\hline $\begin{array}{l}\text { 5.0.7 Risk Reporting } \\
\text { - The sponsor should describe the implemented } \\
\text { quality management approach in the trial and } \\
\text { summarize any important deviations from the } \\
\text { predefined quality tolerance. }\end{array}$ & $\begin{array}{l}\text { 10.1 Improvement } \\
\text { - The organization shall determine any opportunities } \\
\text { and implement any necessary actions to meet customer } \\
\text { requirements and enhance satisfaction. }\end{array}$ \\
\hline $\begin{array}{l}\text { 5.2.2 The sponsor should ensure oversight of any trial- } \\
\text { related duties and functions } \\
\text { behalf. }\end{array}$ & $\begin{array}{l}\text { Organization Roles, Responsibilities, and Authorities } \\
\text { - Ensure that the processes deliver their intended } \\
\text { outputs. }\end{array}$ \\
\hline
\end{tabular}


TABLE NO: 1 - COMPARATIVE STUDY BETWEEN THE INTEGRATED ADDENDUM TO ICHE6(R2) AND QUALITY MANAGEMENT SYSTEM (ISO 9001:2015)

\begin{tabular}{|c|c|}
\hline Integrated Addendum to ICH-EG (R2) & Quality Management System (ISO 9001:2015) \\
\hline $\begin{array}{l}\text { - The intended use of the system } \\
\text { - The potential of the system to affect human subject } \\
\text { protection and reliability. }\end{array}$ & $\begin{array}{l}\text { - Internal issues } \\
\text { - External issues. }\end{array}$ \\
\hline $\begin{array}{l}\text { 5.5.3 }(b) \text { Maintain Standard Operating Procedures } \\
\text { (SOPs) for using these systems. } \\
\text {-The SOPs should cover system setups, installation, } \\
\text { and use. }\end{array}$ & $\begin{array}{l}\text { 8.1 Operational Planning and Control } \\
\text { - The organization shall plan, implement, and control } \\
\text { the processes necessary to meet the requirements. }\end{array}$ \\
\hline $\begin{array}{l}\text { 5.5.3 (h) Ensure the integrity of the data that } \\
\text { describe the extent, structure and context. } \\
\text { - Changes in computerized systems like migration } \\
\text { of data and software upgrade. }\end{array}$ & $\begin{array}{l}\text { 7.5.2 Creating and Updating } \\
\text { (a) Identification and description } \\
\text { (b) } \underline{\text { Format and updating (e.g. language, software }} \\
\text { version) }\end{array}$ \\
\hline $\begin{array}{l}\text { 5.18 MONITORING } \\
\text { 5.18.3 The Extent and Nature of Monitoring: } \\
\text { - The sponsor should develop a systematic, } \\
\text { prioritized, risk-based approach to monitoring } \\
\text { clinical trials. } \\
\text { - Expand the nature of monitoring to permit various } \\
\text { approaches in order to improve the effectiveness } \\
\text { and efficiency of monitoring. }\end{array}$ & $\begin{array}{l}\text { 9.1 Monitoring, Measure, Analysis, and Evaluation } \\
\text { 9.1.3 Analysis and Evaluation } \\
\text { (f) The effectiveness of the actions taken to address } \\
\text { risks and opportunities. } \\
\text { 9.1.1(b) The methods to monitor, measure, analyze, } \\
\text { and evaluate to ensure valid results. }\end{array}$ \\
\hline $\begin{array}{l}\text { 5.18.6 Monitoring Report } \\
\text { (e) Reports of on-site and/or centralized monitoring } \\
\text { should be provided to the sponsor in a timely } \\
\text { manner for review and follow up. } \\
\text { - Result of monitoring activities should be } \\
\text { documented in sufficient detail to allow } \\
\text { verification of compliance with the monitoring } \\
\text { plan. }\end{array}$ & $\begin{array}{l}\text { 9.2 Internal audit } \\
\text { 9.2.1 The organization shall } \\
\text { (a) Plan, establish, implement and maintain an audit } \\
\text { programme(s) including the frequency, method, } \\
\text { responsibilities, planning requirements and reporting, } \\
\text { which shall take into consideration the importance of } \\
\text { processes concerned, changes affecting the } \\
\text { organization, and the results of previous audits. }\end{array}$ \\
\hline $\begin{array}{l}\text { 5.18.7 Monitoring Plan } \\
\text { - The sponsor should develop a monitoring plan that } \\
\text { is tailored to the specific human subject protection } \\
\text { and data integrity risk of the trial. } \\
\text {-The plan should describe the monitoring strategy, } \\
\text { the monitoring responsibilities of all the parties } \\
\text { involved. }\end{array}$ & $\begin{array}{l}\text { 8.1 Operation Planning and Control } \\
\text { - The output of the planning shall be suitable for the } \\
\text { organization's operations } \\
5.2 .1 \text { Customer Focus } \\
\text { (a) Customer and applicable statutory and regulatory } \\
\text { requirements }\end{array}$ \\
\hline $\begin{array}{l}\text { 5.20 Noncompliance } \\
\text { - If noncompliance significantly affects or has the } \\
\text { potential to significantly affect or has the potential } \\
\text { to significant affect human subject protection or } \\
\text { the reliability of trial results is discovered, the } \\
\text { sponsor should perform a root cause analysis and } \\
\text { implement appropriate corrective and prevention } \\
\text { actions. }\end{array}$ & $\begin{array}{l}\text { 10.2 Nonconformity and Corrective Action } \\
\text { When a nonconformity occurs, including } \\
\text { nonconformity arising from any complaints, the } \\
\text { organization shall: } \\
\text { React to nonconformity. } \\
\text { Evaluate the need for actions to eliminate the causes of } \\
\text { nonconformity. } \\
\text { Implement any necessary action. } \\
\text { Corrective action shall be appropriate to the effects of } \\
\text { the encountered nonconformities. }\end{array}$ \\
\hline
\end{tabular}

a.NOTE: The underline phrases represent the direct correlation between the ICH-E6 (R2) and QMS ISO

9001:2015 sentence 


\section{Result}

In the research article, the ISO principles can be compared to clinical trial requirements: focus on customers, provide leadership, involve your people, use a process approach, take a systems approach, encourage continual improvement, get facts before you decide and work with your suppliers [2].

This comparative analysis between ICH-E6 (R2) addendum and QMS ISO 9001-2015 can promote a better understanding of the correlation between the two systems, GCP and QMS. The additional comparative detail as shown in Table 1 will have the potential to contribute to bridging the gap that has not been closed thus far. This presents the common ground for the comparative study between two systems on which to move forward in future approaches.

This directional comparison study is shown in Table 1, and the analysis adopted two comparative approaches: the directional approach and the direct correlation approach. First, the directional approach is a primary approach for comparative analysis between these two systems and we focus the ICH-E6 (R2) guidelines on the QMS ISO 9001-2015 sentences. Example: ICH-E6 (R2) 5.0.3 Risk Evaluation-The sponsor should evaluate the identified risks against the existing risk controls; while in the ISO 9001-2015, we have considered it as 9.1.3 Analysis and Evaluation - The organization shall analyze and evaluate appropriate information arising from measuring or monitoring. The clinical trial process focuses on the risk-based approach, and we need to consider the word risk into consideration with respect to terminology.

Second, the direct correlation approach, as applied to the ICH-E6 (R2) and QMS ISO 90012015 sentences, demonstrates that we can find a sentence/word which allows for a more accurate comparison between the sentences. In this approach, we had further analyzed using sentence-to-sentence correlation and word-to-word correlation.

In sentence-to-sentence correlation, we focus on the conceptual understanding of the sentences in these two systems and find the similarity in meanings. Example: ICH-E6 (R2) 5.18.3 Monitoring to Permit Various Approaches; while in the ISO 9001:2015, we have considered it as 9.1.1(b) methods to monitor.

In word-to-word correlation, we consider the direct comparison between the words in these two systems and find the closest term that can connect the two. This approach will help us achieve a basic understanding between the ICH-E6 (R2) guidelines and QMS ISO 90012015. Example: ICH-E6 (R2) 5.0.4 Risk Control (a) Protocol design, agreement between parties; while in the ISO 9001:2015, we have considered it as 8.3.2 design and development planning methods to monitor; (b) requirements process stages and (d) responsibilities, respectively.

In the Addendum of ICH-E6 (R2) guidelines, a process approach is one that is risk-based: clinical trial processes include consent, randomization, and data processes that are more upto-date: Example: ICH-E6 (R2) 5.5.3 (b) the standard operating procedures should cover system setups, installation, and use; while in the QMS, we have considered it as 8.1 operations shall plan, implement, and control the processes necessary to meet the 
requirements. ICH-E6 (R2) 5.5.3 (h) ensure the integrity of the data; while in the QMS, we have considered it as 7.5.2 Creating and updating (e.g. language, software version).

As this indicates, the above mentions taking a systems approach. The addendum of ICHE6 (R2) guidelines also focuses on monitoring. Studying a direct correlation between the ICH - E6 (R2) and QMS ISO 9001-2015 sentences can help to close any gaps that might exist to achieve quality performance. Example: ICH-E6 (R2) 5.18.3 the extent and nature of monitoring; while the QMS, considers 9.1 monitoring, measure, analysis, and evaluation. This shows that ICH-E6 (R2) takes a risk-based approach to monitor clinical trials. Thus, during the monitoring training or planning, the risk approach should be considered as the main theme for continual improvement.

In this research paper, ICH-E6 (R2) 5.20.1 Noncompliance, when the sponsors team-audit or monitor- discovers noncompliance that significantly affects or has the potential to significantly affect human subject protection or data integrity, the sponsor should conduct a root cause analysis and implement appropriate corrective and preventive actions [1]. The regulatory authorities should take this into consideration when noncompliance is a serious breach of the trial protocol or GCP [8]. For comparison in the ISO 9001-2015, we have considered it as 10.2 Nonconformity and Corrective action. In QMS, the organization shall evaluate the need for actions to eliminate the causes of nonconformity. Corrective action shall be appropriate to the effects of the encountered nonconformities.

This directional comparative study will draw fresh attention to and build practical approaches and methods focusing on risk-based quality management in the clinical trials system. Such a detailed investigation of the role of sponsor, investigator, and trial-site under the risk-based approach, and the monitoring strategy will help us find novel ways to improve the quality of the clinical trials system.

\section{Discussion}

This paper introduces a directional connection between the clinical trial guideline and the quality management system standard, ISO 9001:2015. This approach can give a more accurate comparison and will help find out any missing elements that might be necessary to achieve quality performance during clinical practice.

In this paper, the study involves the integration of the ICH documents and ISO 9001:2015 for the comparative analysis is proceed based on the specific approach that is a directional approach. Firstly, considering the uncommon pathway from the ICH documents towards ISO 9001:2015 which could be the novel approach for the comparative study.

Knowing the objective of the comparative study between two documents is to improve the quality of good clinical practice with the help of the standard documents as ISO 9001:2015. The effectivity of these two comparatives study is that the GCP protocols in Clinical trials are based on the quality outcome and through various studies, the GCP protocols are still not enough for the quality outcome. Whereas, ISO is an implementation standard and this itself is standardized.

Hence, the priority should be given to the GCP protocols during the comparative study. The minimum variation is considered when we are following the directional comparative study. During directional comparative analysis, the initial point (i.e. ICH-E6 (R2) addendum) was taken into an account as crucial information for the minimum information variation. The 
final point of comparative study which is QMS ISO 9001-2015 and relevant information related to the initial point is to be considered for the comparative study. Furthermore, if necessary, the QMS ISO 9001-2015 protocols can be improvised and modified which could be suitable for the ICH-E6 (R2) addendum. Hence, the ICH documents for the quality improvement is based on the reference standard that is ISO 9001:2015 for the comparative study and through directional approach.

This approach which helps to improve the quality of other system documents. But the directional approach is much related to the effective comparative study. This pathway can help to find the missing elements and help to develop the novel fundamental criteria during the comparative study. Therefore, this paper considering the directional comparative study for improvement of the clinical trial system could be a novel approach for analysis.

As a result, it is possible that we can achieve a more productive approach using the uncommon path of comparative analysis between the clinical trial guideline and the QMS. Such an analytical approach has not been studied to date. The pharmaceutical industry is highly regulated and has a firmly rooted quality system. Therefore, by analyzing and directing quality efforts on a long-term basis, the ISO 9001: 2015 international standard could act as a clinical trial framework for quality management without any loss of compliance. The addendum also focuses on risk-based approaches and has updated and implemented the GCP guidelines based on process and system reviews. Thus, clinical research and trial systems have moved towards quality performance based on a novel approach.

In their pursuit of excellence, pharmaceutical companies attempt to obtain a quality certification, namely ISO 9000 accreditation. Certification is attractive as a means of impressing new customers and convincing existing ones that they are dealing with a company that is continually seeking improvement [7]. By comparing the clinical trial guidelines and the ISO 9001:2015, it may be possible to identify the missing elements that might be crucial for quality performance to the clinical trial system.

As conceptual understanding may vary among individuals, the conflict in the conceptual and terminological stability between the two systems is one of the major problems faced during the comparative study. To address this, we have devised new approaches to evaluate the studies. This paper generally focuses on the directional and direct correlation approaches. Our advanced approach focuses more on finding the word-to-word / sentence-to-sentence similarities between ICH-E6 (R2) addendum and QMS ISO 9001-2015.

In considering the Annex SL for a broader understanding of comparative study in the addendum of ICH-E6 (R2) guidelines, it might help to consider the words or sentences in Annex SL as a base for further comparative analysis between these two systems. For the deeper study, we can consider the repetitive words or definition as one of the criteria for the analysis. In addition, we can use the repeating word frequency analysis, for example, tf-idf (term frequency-inverse document frequency) for information retrieval or text mining.

Measuring the similarity between the words, sentences, and paragraphs in the ICH guidelines documents and ISO 9001-2015 is a good systematic approach for the comparison through automatic scoring and easy information retrieval, for better understanding and analysing these systems.

The knowledge-based similarity is one of the semantic similarity measures that seek to identify the degree of similarity between words using information derived from semantic 
networks [9]. Through this, we can derive the common usage of words which can be grouped together, and make a conceptual interlink between the words, sentences, or paragraphs of these two systems. Thus, we can develop our own ground rules for future information analysis which can be applicable in another system.

Semantic similarity is a kind of relatedness between two words. It covers a broader range of relatedness between concepts that include extra similarity relations such as is-a-kind-of, isa-specific-example-of, and is-a-part-of [9]. We can use this kind of comparative study to arrive at better ground rules and build strong coordination between the GCP guidelines and ISO 9001-2015. With the semantic similarity and repeating word frequency analysis, we can gain information, which we can use not only for comparative analysis of these two systems but also for another system.

The directional comparative approach from the clinical trial guidelines (ICH E6 (R2)addendum toward the ISO 9001-2015 quality standard gives more weight to the clinical trial guidelines. By measuring the similarities, we need to consider the directional approach as a baseline concept that shouldn't deviate from the original meaning in the clinical trial guidelines. Hence, we should focus more on the quality standard to find other alternative sentences/paragraphs which give greater similarity than the current compared sentences/paragraphs.

The comparative analysis based on the directional approach allows us to calculate the percentage similarity between ICH-E6 (R2) addendum and QMS ISO 9001-2015. The directional approach-based calculation gives preference to the start point of the comparative analysis. Hence, in the future, we can develop a fundamental rule for a comparative study between these two systems or more broadly, between any two systems.

\section{Conclusion}

The analysis of the Integrated Addendum to ICH-E6 (R1), guidelines GCP-ICH-E6 (R2) with the Quality Management System ISO 9001: 2015 shows that the paragraphs in the good clinical practices (GCP) guideline have a connection with the paragraphs in the ISO 9001:2015.

Since clinical trial practice involves the participation of human subjects, it is highly regulated. Also, its quality management system practices are firmly rooted in the clinical trial system. However, there is still a conflict in the conceptual and terminological differences between the GCP guidelines and ISO 9001:2015 quality management methodologies. The GCP guidelines provide updated information and additional addendum through a review of the system and process. As shown, it is crucial that GCP guidelines move toward this approach to quality performance. Even though clinical trials are conducted under the GCP guidelines and ISO standard to ensure the safety of the patients, GCP guidelines protocols are too narrow in focus and QMS ISO 9001:2015 methodologies are too wide to understand. The drawback that was found during the directional approach comparative studies is essential for developing the fundamental criteria for the effective comparative study. The directional comparative study between the GCP guidelines and ISO in this analysis can be used to close the gap and find new approaches for quality improvement in the clinical trial system.

With the help of different information retrieval processes, we can extract information for better analysis of these two systems. By finding the degree of similarity between the GCP 
guidelines and ISO, we can trace the fluctuation in the expression of information. Also, analysing word frequency presents a way forward with better, more accurate comparisons that we can utilize in future analysis.

This domain of a directional comparison analysis can be the preparatory step for developing novel approaches for comparative information analysis between the clinical trial guidelines (ICH E6 (R2)-addendum) and the ISO 9001:2015 quality standards. Hence, through directional comparative analysis with consideration of the degree of similarity and repetitive word frequency between these two systems, this study can create novel approaches which can establish ground rules for the comparative study between any two systems.

\section{Conflict of interest}

There are no conflicts of interest.

\section{References}

[1] "International council for harmonization of technical requirements for pharmaceutical for human use (ICH) Harmonization Guideline, Integrated Addendum to ICH E6 (R1): Guideline for Good Clinical Practice E6 (R2)," current Step 4 version, dated 9 Nov 2016.

[2]“ISO (2015), International Standard ISO 9001:2015 Quality Management Systems Requirements,” JIS Q 9001:2015.

[3]Elizabeth Murry, "A comparative analysis of quality management standards for contract research organizations in clinical trials," International Journal of Health Care Quality Assurance, vol. 20 no. 1, 2007, pp. 16-33.

[4] V. Ye. Dobraova, K. L Ratushna, Ie. F. Grintsov and N. P. Bezugla, "The comparative analysis of the ISO 9001: 2015 standard and good clinical practice guideline: the framework for improving management of clinical trials," National University of Pharmacy, vol. 21 no. 2, 2017, pp. 4-9.

[5] "ICH reflection on GCP Renovating: Modernization of ICH E8 and subsequent renovation of ICH E6", Jan 2017.

[6]F. Sweeney, "Merging GCP and ISO 9000 requirements -a source of synergy in quality management of clinical research", Drug Information Journal, Vol. 28, 1994, pp. 1097104.

[7] Rodney McAdam, Niger Barron, "The role of quality management in pharmaceutical development: clinical trials analysis," International Journal of Health Care Quality Assurance, vol. 15 no. 3, 2002, pp. 106-123.

[8] A. Bhatt, "International Council for harmonization E6(R2) addendum: Challenges of implementation," Perspectives in Clinical Research, vol. 8 no. 4, 2017, pp. 162-166.

[9] Mihalcea, D., Corley, C. \& Strapparava, C. "Corpus based and knowledge-based measures of text semantic similarity." In Proceedings of the American Association for Artificial Intelligence, Boston, MA, 2006, pp. 775-780 